\title{
Electromagnetic interaction between Jupiter's ionosphere and the Io plasma torus
}

\author{
Yi-Jiun Su $\dagger$ \\ Department of Physics, the University of Texas at Arlington, Arlington, TX 76019, USA \\ email: yijiunsu@gmail.com
}

\begin{abstract}
The electromagnetic interaction between Jupiter and Io has been studied extensively since the discovery of Io-controlled decametric radio emissions (DAMs). A variety of mechanisms for electromagnetic disturbances have been considered including a unipolar inductor, the excitation of large-amplitude Alfvén waves, the generation of electrostatic electric fields parallel to the ambient magnetic field, and etc. Recently, three auroral acceleration regions categorized by terrestrial physicists have been applied to the Jupiter-Io coupling system: the Alfvénic acceleration region is associated with bright emissions at Io's magnetic footprint, whereas the quasi-static system of anti-planetward and planetward currents set up at the inner and outer edges of the torus in the downstream region of Io's wake. This review paper summarizes the current understanding of the coupling mechanisms between Jupiter's ionosphere and the Io plasma torus, as well as the electron acceleration mechanism necessary to excite Io-associated emissions.
\end{abstract}

Keywords. Acceleration of particles - waves - plasmas - planets and satellites

\section{Introduction}

The electrodynamic interaction of the Jupiter-Io system is unique in our solar system particularly due to the fact that (1) the strong magnetic field of Jupiter creates the largest magnetosphere, (2) Jupiter is the fastest rotating planet, and (3) Io is the most volcanically active moon (Bagenal 2007; Saur et al. 2004). Unlike the terrestrial electromagnetic dynamo primarily driven by the solar wind, the electrodynamics of Jupiter are dominated by the planet's fast spin with a 10-hour rotational period. The closest Galilean moon to Jupiter - Io, located at 5.9 Jovian-centric distance $\left(R_{J} \approx 71,500 \mathrm{~km}\right)-$ is embedded deep within its vast magnetosphere, which has been known to extend to the orbit of Saturn.

The dominant neutrals generated by volcanoes on Io are sulfur and oxygen (1-3 ton $s^{-1}$ ), most likely in the form of $\mathrm{SO}_{2}$. Approximately, 1/3-1/2 of the neutral atoms are ionized by photo-ionization, electron impact ionization, and charge exchange, thereby adding heavy ions, such as $O^{+}, S^{++}, S^{+}, O^{++}$, and etc., to the inner magnetosphere, where they spread out into a donut shape encircling Jupiter known as the Io plasma torus. The newly ionized neutrals are referred to as 'pickup ions' because as soon as they are ionized, they are accelerated to the speed of the bulk plasma flow, $74 \mathrm{kms}^{-1}$. Io orbits Jupiter with a velocity of $17 \mathrm{kms}^{-1}$, so the plasmas flow over the moon with a relative velocity of $57 \mathrm{kms}^{-1}$ ahead of Io in its orbital motion. This flow of magnetized plasmas sweeps through the obstacle, Io, producing intense electrodynamic interaction (Kivelson et al. 2004, and references therein). The plasma density, momentum, and energy are modified locally through elastic and inelastic collisions with Io's atmosphere and ionization processes. This interaction also extends far away towards Jupiter's ionosphere, particularly along the magnetic

$\dagger$ Now at Air Force Research Laboratory/RVBXP, 29 Randolph Road, Hanscom, AFB, MA 01731, USA 
field line. Auroral emissions observed at the magnetic footprint of Io and its wake or trail emissions are evidence of such interaction. An example of a Jovian auroral image taken by the Hubble Space Telescope is presented in the top panel of Figure 1 (Clarke et al. 2002).

\subsection{Early theories}

The electromagnetic interaction between Jupiter and Io has been investigated extensively since the first discovery of Iocontrolled DAMs by Bigg (1964). One of the early theories describing the JupiterIo interaction was proposed by Piddington \& Drake (1968) and Goldreich \& Lynden-Bell (1969) and was referred to as the unipolar inductor model: fieldaligned currents link Io to Jupiter's ionosphere through magnetospheric plasmas. A schematic representation of the current loop is similar to that shown in the bottom panel of Figure 1. A $J \times B$ force is generated to accelerate plasmas downstream of Io to the corotation speed (into the page of the bottom panel). Because Jupiter's magnetic field points southward at the torus, a radially outward current at Io completes the circuit while field-aligned currents flow anti-planetward and planetward at Io's inner and outer edges, respectively. Based on the unipolar inductor theory, the induced electric potential difference between the outer and inner boundaries of Io was estimated to be $415 k V \approx u(57$ $\left.k \mathrm{~ms}^{-1}\right) \times B(2,000 \mathrm{nT}) \times R_{\text {Io }}(3,636 \mathrm{~km})$. However, the assumption of an extremely high conductivity at Io resulting in frozenin Jovian magnetic fields with Io was not realistic.

Electric and magnetic disturbances are generated when a flux tube of magnetospheric plasmas passes through the Io plasma torus. Due to the relative motion ( $u$ ) between Io and the corotating magnetospheric plasmas, electromagnetic perturbations are propagated away from the torus with an angle $\theta_{A}=\tan ^{-1}\left(u / V_{A}\right)$, where $V_{A}$ is the Alfvén speed. This bent magnetic field structure is known as the Alfvén wing (Drell et al. 1965; Goertz \& Deift 1973; Goertz 1980; Neubauer 1980; Southwood et al. 1980). In spite of the exact nature of the
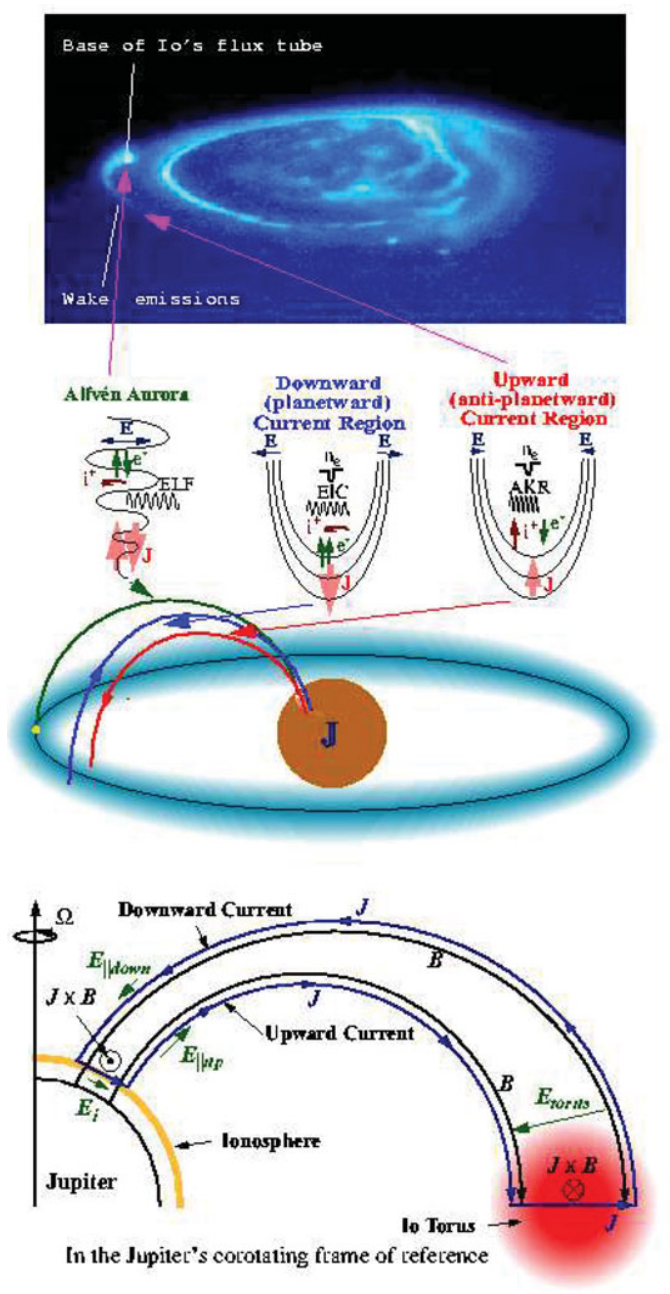

Figure 1. The top panel shows a Jovian auroral image, where the Io-induced aurora is seen on the left with the brightest emissions at the base of the Io flux tube and an emission trail extending downstream. A depiction of the three types of auroral regions is shown in the middle panel, where the green, blue, and red lines represent the Alfvénic acceleration region, the planetward current region, and the anti-planetward current region, respectively. The quasi-static current structure downstream of Io's wake in Jupiter's corotating frame is illustrated in the bottom panel (after Su et al. 2003). 
perturbation in Io's vicinity, energy is carried away in the form of an Alfvén wave. Due to the density gradient of the plasma torus, a substantial amount of the wave energy may be reflected back into the torus. Exactly how much energy escapes from the Io torus remains a topic of debate.

Several major unsolved questions involving the Jupiter-Io interaction were summarized by Saur et al. (2004), including the coupling mechanisms between Io and Jupiter's ionosphere and the electron acceleration mechanism necessary to excite Io-associated emissions. This paper describes the author's perspective in addressing both questions.

\subsection{Recent theories}

Motivated by Jovian auroral images (Clarke et al. 2002; 2004), Delamere et al. (2003) categorized the Io-Jupiter interaction into three phases: (1) an initial mass-loading interaction; (2) the plasma acceleration in the wake of Io; and (3) the steady-state decoupling. The first phase is dominated by the combination of collision-dominated flows and mass loading processes with a time scale of $\sim 60 \mathrm{sec}$. The second acceleration phase is due to the momentum transfer through Alfvénic disturbances with a time scale of $\sim 8 \mathrm{~min}$. Delamere et al. (2003) estimated that only $\sim 20 \%$ of the input momentum is transmitted to high latitudes due to the internal reflection of the torus density gradient. Once Alfvén waves have bounced between Io and Jupiter's ionosphere a few times, quasi-static fieldaligned currents are set up at Io's wake during the third phase. In this final phase, the plasma velocity is $\sim 1 \mathrm{kms}^{-1}$ deviated from the corotation speed due to the magnetic decoupling rather than the rigid corotation. From observations of trail emissions, this decoupling effect can last as long as 5 hours corresponding to $180^{\circ}$ in longitude.

Mauk et al. (2002) indicated several similarities between the Earth and Io's auroras. Despite the complexity of auroral observations, terrestrial auroral physicists have simply classified the auroral acceleration process into three types based on distinct characteristics of particle and field measurements: (1) the upward current region; (2) the downward current region; and (3) the Alfvénic acceleration region (Paschmann et al. 2003, Chapter 4). These regions are illustrated below the auroral image in Figure 1. The prominent features of the quasi-static upward current region are up-going field-aligned ions and downgoing accelerated electrons, narrowly peaked in energy but broad in pitch angle. This region consists of multiple inverted-V electron structures which are associated with converging electric fields and low-density cavities. In the downward current region, upward field-aligned electron beams are associated with diverging electric fields. The downward current region is more dynamic and complex than the upward current region. It is often combined with Alfvénic fluctuations and is rarely associated with detectable auroral emissions. At Earth, the Alfvénic acceleration region is observed near the open-closed field line boundaries which map to reconnection regions on the dayside magnetopause and on the nightside magnetotail. The most distinctive features are counter-streaming electron bursts, enhanced ion conics, and the filamentary nature of currents generated by the propagation Alfvén waves rather than quasi-static potential structures. The ratio of $\delta E / \delta B$ is approximately equal to the local Alfvén speed ( $\mathrm{Su}$ et al. 2001).

Although Jovian auroral observations are currently limited to remote imaging, fundamental physics learned from Terrestrial studies should be universally applicable to any magnetized planet system. Su et al. (2003) proposed the three auroral acceleration processes described above for the Earth to be active magnetosphere-ionosphere coupling mechanisms on Jupiter as well (see the middle panel of Figure 1). The most intense counter-streaming electron fluxes are driven by propagating Alfvén waves associated with oscillating field-aligned electric fields (Chaston et al. 1999). Therefore, the bright emission at the Io magnetic footprint is caused by Alfvén dominated precipitation, whereas 
quasi-static currents are set up in the downstream region of Io's wake. An analogy between the Earth's midnight auroral zone during a substorm and the Jupiter-Io interaction was summarized in an illustration by Ergun et al. (2006). Recent theoretical studies of the upward (anti-planetward) current region are discussed in $\S 2$ followed by a description of the physical processes of the Alfvénic acceleration region in $\S 3$.

\section{The quasi-static upward current region}

The fundamental physics of the Earth's upward current region is well established, however, application of this knowledge to other magnetized planets has been limited. The aurora is generated by magnetospheric electrons slamming into the atmosphere and exciting the airglow. These precipitating electrons carry the upward field-aligned current. Due to the magnetic mirror, the current density is limited by the amount of electrons inside the loss cone. Observations have provided compelling evidence that this acceleration process is achieved by parallel electric fields, which act to accelerate electrons downward pushing more current carriers into the loss cone. Illustrations of electron distributions in three regimes of the current-voltage relation are shown in Figure 2.

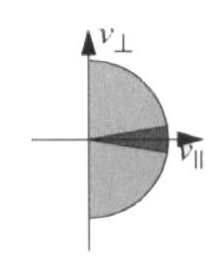

Thermal flow $\mathrm{e} \Phi_{\|} \ll 1$

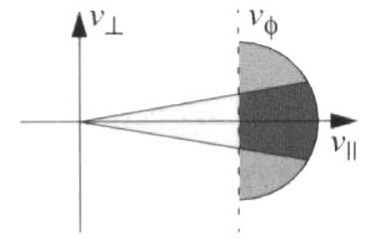

Linear regime $1 \ll \mathrm{e} \Phi_{\|} \ll \mathrm{R}_{\mathrm{M}}$

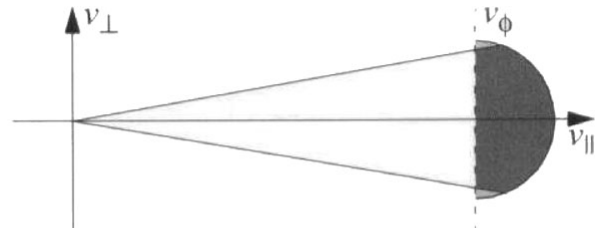

Saturation

$R_{M} \ll e \Phi_{\|}$

Figure 2. The thermal flow is restricted to a small region of phase space (left). Increasing $v_{\|}$ due to $e \Phi_{\|}$allows more electrons to flow into the ionosphere (middle) until the current saturates (right). Dark shaded areas within the loss cone contain precipitation electrons which carry an upward current (after Paschmann et al. 2003).

This theoretical relationship was originally recognized by Knight (1973) and summarized by Paschmann et al. (2003) and Ergun et al. (2008).

$$
\begin{aligned}
J_{\| \text {Iono }} & =J(z)\left\{R_{M}(z)-\left(R_{M}(z)-1\right) \exp \left[-\frac{e \Phi_{\|}}{k_{B} T_{e}(z)\left(R_{M}(z)-1\right)}\right]\right\}, \\
J(z) & =\operatorname{en}_{e}(z) \sqrt{\frac{k_{B} T_{e}(z)}{2 \pi m_{e}}}, \quad R_{M}(z) \frac{B_{\text {Iono }}}{B(z)}
\end{aligned}
$$

where $J_{|| \text {Iono }}$ represents the parallel current density at the ionosphere, while $n_{e}(z), T_{e}(z)$, $J(z)$, and $R_{M}(z)$ are electron density, electron temperature, electron thermal current, and the magnetic mirror ratio, respectively, at a location $z$ between the ionosphere and magnetosphere. The electron thermal current should be at its minimum at $z$. $\Phi_{\|}$is the parallel electrostatic potential between ionosphere and $z$.

On an auroral flux tube at Earth, $J(z)$ is at its minimum in the plasmasheet. For the typical density $\left(0.2 \mathrm{~cm}^{-3}\right)$ and temperature $(1 \mathrm{keV})$ of plasmasheet electrons, the currentvoltage relations for mirror ratios of 400 and 1000 are plotted as the solid and dashed lines, respectively, while the linear Knight relation is represented by the dotted line in 

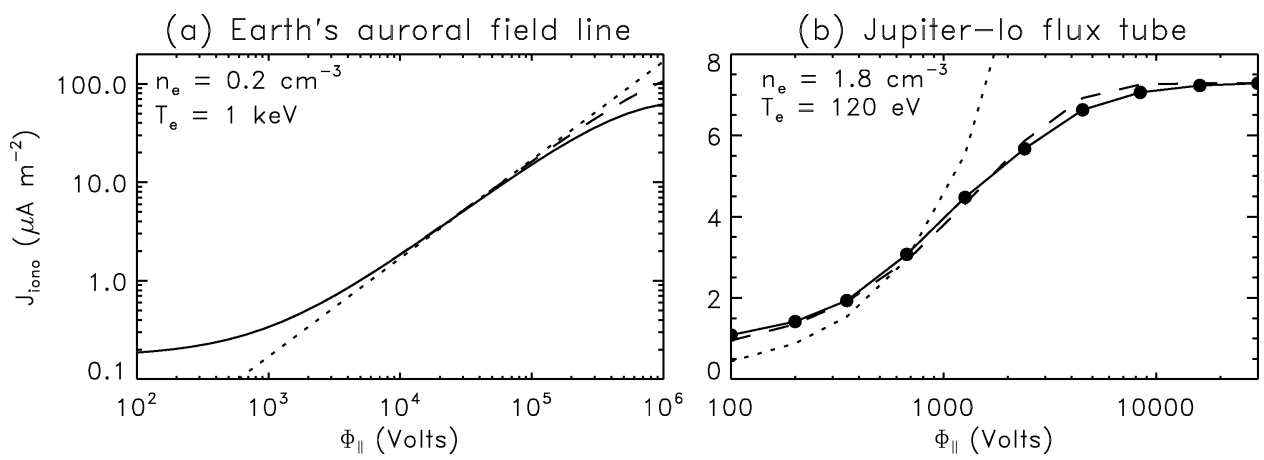

Figure 3. The current-voltage relation (a) at Earth and (b) on the Jupiter-Io flux tube.

Figure 3(a). The majority of observed data fall within the linear regime, hence, a linear Knight relation $\left(J_{\text {Iono }}=K \Phi_{\|}\right.$, where $\left.K=e^{2} n_{e} / \sqrt{2 \pi m_{e} k_{B} T_{e}}\right)$ is a good approximation for the upward current region of Earth's auroral field lines.

At Jupiter, $J(z)$ occurs near the minimum of the combination of gravitational and centrifugal potentials, $\sim 2.5 R_{J}$ from Jupiter's center ( $\mathrm{Su}$ et al. 2003). It is very important to note that the plasma parameters at $2.5 R_{J}$ are dramatically different from those in the Io torus, because the majority of Iogenic heavy ions are confined within the torus due to Jupiter's strong centrifugal force. In-situ measurements of plasma parameters at high latitudes are currently unavailable due to the lack of satellite missions in Jupiter's polar region. Su et al. (2003) have self-consistently obtained plasma parameters along the Jupiter-Io flux tube by utilizing a quasi-static Vlasov code modified from an Earth's model (Ergun et al. 2000a). The basic idea of the code is to solve the Poisson's equation along the field line by specifying phase-space distributions for various species at boundaries. The lower boundary conditions for the ionospheric parameters are based on radio occultation measurements (Fjeldbo et al. 1975; 1976; Eshleman et al. 1979; Hinson et al. 1997), while the upper boundary conditions for the torus parameters are obtained from Voyager (Bagenal 1994) and Galileo observations (Crary et al. 1998).

The current-voltage relation on the Jupiter-Io flux tube is shown as the solid line with solid circles in Figure 3(b) based on the fixed boundary parameters listed in Table 1. The upper limit $(32 \mathrm{kV})$ of the parallel potential in Figure 3(b) was estimated from the mean electron energy at $30^{\circ}$ downstream of Io based on the Space Telescope Imaging Spectrograph combined with a Jovian atmosphere model (Gérard et al. 2002). More recent analysis suggests that downstream mean energies may be in the range of $100 \mathrm{~s} \mathrm{eV}$ (Gérard, private communication). The lower limit of the x-axis is, therefore, set at 100 $V$. In addition, the theoretical current-voltage relation (Eq. 2.1) and the linear Knight relation are plotted as the dashed and dotted lines, respectively, based on the electron

Table 1. Boundary conditions of Figure 3(b)

\begin{tabular}{lccc}
\hline Species & $\mathrm{N}\left(\mathrm{cm}^{-3}\right)$ & $\mathrm{T}(\mathrm{eV})$ & Boundary \\
\hline Ionosphere $H^{+}$ & $2 \times 10^{5}$ & 0.31 & lower \\
Ionosphere $e^{-}$ & $2 \times 10^{5}$ & 0.31 & lower \\
Io $O^{+}$ & 1750 & $T_{\|}=35, T_{\perp}=70$ & upper \\
Io $S^{+}$ & 250 & 50 & upper \\
Io $e^{-}$ & 2000 & $5 \mathrm{eV}^{a}$ & upper \\
\hline
\end{tabular}

${ }^{a}$ a kappa distribution $(\kappa=3)$ 
density $\left(1.8 \mathrm{~cm}^{-3}\right)$ and temperature $(120 \mathrm{eV})$ at $\sim 3 R_{J}$ with the mirror ratio of 13.8 . When the potential is greater than $2 k V$, it apparently violates the linear Knight relation because the upward current approaches saturation (Ray et al. 2008; Su et al. 2003). The ionospheric current density increases with increasing $O^{+}$density and/or parallel temperature (not shown here) because additional magnetospheric electrons are required to balance the ion density at high latitudes to satisfy the quasi-neutrality condition. Moreover, the majority of the upward current is carried by the hotter population of electrons ( $\mathrm{Su}$ et al. 2003).

Although proton measurements in the immediate vicinity of Io have been reported by Frank \& Paterson (1999) and Chust et al. (1999), it is difficulty to separate them from the high-density heavy ions in the plasma torus. Su et al. (2003) explored the possibility of $\mathrm{H}^{+}$imposed at the upper boundary and concluded that $H^{+}$ions became the major species at 2-3 $R_{J}$ as the light ions are able to easily escape the strong centrifugal confinement of the torus. Therefore, the ionospheric upward current density carried by precipitating electrons increases with increasing $H^{+}$density and temperature. Based on auroral images at $10^{\circ}-20^{\circ}$ downstream of Io's wake, Gérard et al. (2006) reported $\sim 10 k R$ ultraviolet emission corresponding to an energy flux of $1 \mathrm{mWm}^{-2}$ resulting in an ionospheric current density of approximately $1 \mu \mathrm{Am}^{-2}$ assuming the energy of the accelerated electrons to be $1 \mathrm{keV}$. This rough estimation suggests that the $H^{+}$density should be much less than $1 \mathrm{~cm}^{-3}$ at high latitudes.

\section{The Alfvénic acceleration region}

The existence of Alfvén waves near Io have been established by Voyager 1 and Galileo (Saur et al. 2004, and references therein), however, the amount of wave energy escaping the torus is not well understood. Additionally, scientists do not know precisely where the wave energy is converted into electron energy to power the Io auroral spot. The majority of numerical studies are based on the magnetohydrodynamic (MHD) theory (e.g., Wright 1987; Dols 2001). In this paper, the propagation of dispersive Alfvén waves along the Jupiter-Io flux tube, as well as electron acceleration by these waves, are discussed in $\S 3.1$.

Less than $10 \%$ of DAMs were observed to exhibit a distinct feature of discrete short pluses, known as S-bursts (Zarka 1992, and reference therein). S-bursts are strictly associated with an Io-dependent emission source mostly from the Io-B region in the Jupiter Central Meridian Longitude (Ergun et al. 2006). The generation mechanisms of S-bursts are important elements in understanding the Jupiter-Io system and are discussed in $\S 3.2$.

\subsection{Dispersive Alfvén waves}

In the ideal MHD theory, Alfvén waves have no field-aligned electric field component and therefore provide no parallel acceleration to particles. A parallel electric field is generated by dispersive Alfvén waves when the parallel electric force is balanced by the electron inertia, electron pressure gradient, and/or the finite ion gyro-radius effect (Stasiewicz et al. 2000, and references therein). A dynamic model including the dispersive effects should be considered to properly address science questions associated with the Alfvénic acceleration region ( $\mathrm{Su}$ et al. 2004; 2006). The general dispersion relation of dispersive Alfvén waves was summarized recently by Jones \& Su (2008) as

$$
v_{D A W}^{2}=\frac{\omega^{2}}{k_{\|}^{2}}=v_{A}^{2} \frac{1+k_{\perp}^{2} \rho^{2}}{1+k_{\perp}^{2} \lambda_{e}^{2}} ; \quad \rho^{2}=\rho_{i}^{2}+\rho_{s}^{2}
$$

where $\lambda_{e}=\sqrt{m_{e} / n_{e} e^{2} \mu_{o}}, \rho_{s}=\sqrt{m_{i} T_{e}} / e B$, and $\rho_{i}=\sqrt{m_{i} T_{i}} / e B$ are the electron skin depth, ion acoustic gyro-radius, and ion gyro-radius, respectively. $v_{A}$ is the ideal Alfvén 
speed. When plasma $\beta>m_{e} / m_{i}$, the kinetic effect is the dominant component including the ion gyro-radius effect and the electron pressure gradient. On the other hand, the electron inertial effect becomes important when $\beta<m_{e} / m_{i}$. The parallel electric field changes polarity as dispersive Alfvén waves pass through the transition region $(\beta=$ $\left.m_{e} / m_{i}\right)$. The real part of the parallel electric field reduces to zero, therefore, no particle acceleration occurs at this point. At Earth, the transition region is located at $\sim 4-5 R_{E}$. On the Jupiter-Io flux tube, this transition region is situated within the Io plasma torus.

Crary (1997) proposed that electrons gain energy near the edge of the torus by Fermi acceleration through parallel inertial Alfvén electric fields, hence, the wave itself carries virtually no energy to the ionosphere. Das \& Ip (2000) observed kinetic Alfvén waves near Io and suggested that these waves were responsible for electron acceleration within the torus. However, Jones \& Su (2008) argued that the phase speed of the dispersive Alfvén wave is too small to account for the resonant acceleration near the torus, hence, it is too weak to cause the observed bright Io spot at the magnetic footprint (Gérard et al. 2002). Therefore, Jones \& Su (2008) suggested that electron acceleration occurs in the low density region $\left(\leqslant 4 R_{J}\right)$ outside the Io plasma torus. Moreover, the maximum $E_{\|}$ is located at $\sim 1.5 R_{J}$, which can be two orders of magnitude higher than $E_{\|}$at $6 R_{J}$ along the Jupiter-Io flux tube.

According to the above arguments, a certain amount of wave energy should be able to escape from the torus and exchange its energy with particles in the region of tenuous plasma. By utilizing a 1D MHD code, Wright (1987) and Dols (2001) suggested $\sim 25 \%$ and $40 \%$, respectively, of the Alfvén wave power leaving the torus. With a 2D fluid model, Delamere et al. (2003) indicated that $\sim 20 \%$ of the input momentum is transmitted to ionosphere. Based on simulation results from a 1D linear gyro-fluid model including the dispersive effect of Alfvén waves, $\mathrm{Su}$ et al. (2006) showed that the first major reflection

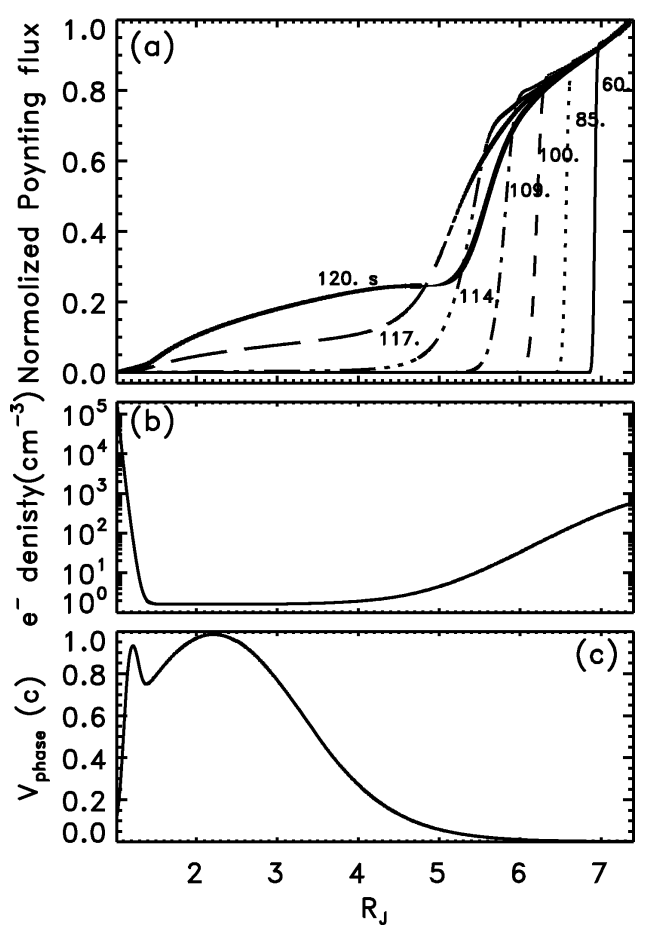

Figure 4. The simulation result based on the gyro-fluid model. (a) Normalized time-integrated Poynting flux at various times. (b) The electron density profile. (c) The phase speed of the dispersive Alfvén wave (after $\mathrm{Su}$ et al. 2006). occurs at $\sim 5.5 R_{J}$ from Jupiter due to the torus density gradient (Figure $4 \mathrm{~b}$ ), while $\sim 20 \%$ of the wave energy reaches $3.5 R_{J}$ (Figure $4 \mathrm{a})$. A second reflection occurs at the peak of the Aflvén speed ( $\sim 2.3 R_{J}$ in Figure $\left.4 \mathrm{c}\right)$. The majority of low-frequency, long-wavelength waves were unable to reach the Jupiter's ionosphere without wave breaking, phase mixing, or other nonlinear processes, however, a significant energy flux may be transferred via high-frequency, small-wavelength waves to the ionosphere.

\subsection{Generation mechanisms of S-bursts}

Wu \& Lee (1979) were the first to explain the generation of terrestrial auroral kilometric radiation $(\mathrm{AKR})$ by suggesting a loss-cone instability with a weakly relativistic 
treatment, which was coined the electron-cyclotron maser by Melrose \& Dulk (1982). The same mechanism was applied to explain DAM from Jupiter, as well as analogous radiations from other magnetized outer planets (Zarka 1992). Radiations excited near the local electron cyclotron frequency are amplified through a gyro-resonant interaction from a perpendicular-driven instability $\left(\partial f / \partial v_{\perp}>0\right.$, where $f$ is the electron phase-space distribution function). In the 1990s, an important modification was made to the original maser theory that strong auroral radiations are driven by a shell (or horseshoe) distribution in the auroral density cavity due to quasi-static parallel electric fields (Ergun et al. 2000b; 2002), because the loss-cone maser does not provide sufficient energy to power AKRs. The electron distributions responsible for the maser instability are illustrated in Figure 5. Although the maser theory explains the observed radiation frequency occurring at the local cyclotron frequency, it is unable to interpret the periodicity of S-bursts.
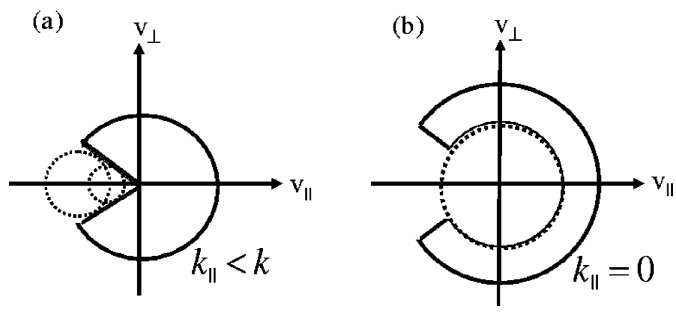

Figure 5. Schematic illustration of electron distributions for (a) the loss-cone maser and (b) shell maser, where the dotted circle represent resonance circles. (after Su et al. 2007)

Ergun et al. (2006) suggested that the S-burst periodicity is associated with the eigenfrequency of the ionospheric Alfvén resonator, $v_{A I} / 2 \pi H_{P}$, where $v_{A I}=B / \sqrt{\mu_{o} \rho}$ and $H_{p}=k_{B} T / m g$ are the Alfvén velocity at the ionosphere and the ionospheric scale height, respectively (Lysak 1991). By using the gyro-fluid model, Su et al. (2006) demonstrated that the inertial Alfvén wave originating in the torus bounces between the ionosphere and the location of the first peak of Alfvén phase velocity (at $1.2 R_{J}$ in Figure 4c). After taking a fast Fourier transform of simulated wave forms, the fundamental eigenfrequency and higher harmonics of the resonator (few-100 Hz) were found to be comparable to observed reoccurrence frequencies of S-bursts. Moreover, the eigenfrequency decreases with increasing ionospheric density and with increasing scale height. For example, with an ionospheric density of $2 \times 10^{5} \mathrm{~cm}^{-3}$ and a scale height of $500 \mathrm{~km}$, Su et al. (2006) found the fundamental eigenfrequency of the resonator to be $\sim 20 \mathrm{~Hz}$, comparable to the most probable reoccurrence rate of S-bursts. Ergun et al. and $\mathrm{Su}$ et al. (2006), therefore, suggested the ionospheric Alfvén resonator as the likely driver explaining multiple occurrences of S-bursts.

The Alfvén wave producing the ionospheric resonator is also responsible for electron acceleration ( $\mathrm{Su}$ et al. 2007). Unstable electron distributions, such as shell, ring, or conics, are generated by parallel electric fields associated with this inertial Alfvén wave ( $\mathrm{Su}$ et al. 2007; Hess et al. 2007; 2008). As stated in the maser theory, unstable electron distributions are the source of remotely observed auroral radiation with a condition that the plasma frequency is much less than the electron cyclotron frequency $\left(\omega_{p e} / \omega_{c e}<<\right.$ 1), however, in-situ observations are currently unavailable near the ionosphere of the Jupiter-Io flux tube to confirm the Alfvén-driven maser hypothesis. Su et al. $(2007 ; 2008)$ supported the Alfvén-driven maser theory as the generation mechanism of S-bursts on the basis of Earth-based satellite measurements.

Although rare, Su et al. (2008) found eight S-burst events in the Earth's Alfvénic acceleration region from three years of FAST data. An example of Earth-based S-bursts is displayed in Figure 6, while observed electron distributions associated with each of the 8 events are shown in Figure 7. Since no event was found when the satellite passed directly through the emission source region, the ratio of $\omega_{p e} / \omega_{c e}$ was not able to be precisely determined. However, all events were found near apogee of the FAST orbit in the midnight local 
time sector during winter months suggesting a preference for low plasma density conditions. At Jupiter, the condition for the weakly relativistic cyclotron maser instability is easily satisfied above a few thousand $\mathrm{km}$ up to $4 R_{J}$ due to Jupiter's strong magnetic field (Ergun et al. 2006), which

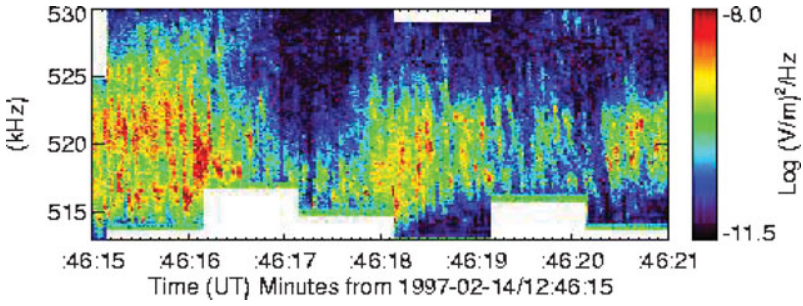

Figure 6. Earth-based S-burst (after Su et al. 2008). explains the higher occurrence rate of Jovian S-bursts.

The reoccurrence frequency of Earth-based S-bursts was found to fall between 7 and $18 \mathrm{~Hz}(\mathrm{Su}$ et al. 2008) similar to that of Jovian S-bursts, but an order of magnitude higher than the typical frequency $(\sim 1 \mathrm{~Hz})$ of Earth's ionospheric Alfvén resonator (Lysak 1991). A higher reoccurrence rate requires a smaller ionospheric scale height and/or a lower ionospheric density than typically observed. Based on simulation results from a gyro-fluid code with a test particle scheme, $\mathrm{Su}$ et al. (2008) proposed multiple Alfvénic disturbances at the upper boundary (i.e., the Io tours at Jupiter and the magnetotail at Earth) as another possible driver for the S-burst periodicity. Each discrete S-burst radiation is generated when unstable electron distributions due to each Alfvén wave pulse passes through regions with conditions matching the maser instability.

(a) A single shell

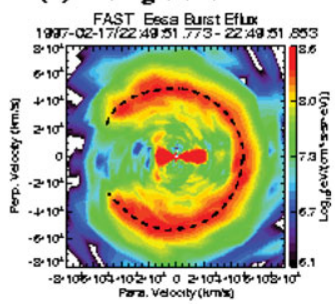

(e) Conic

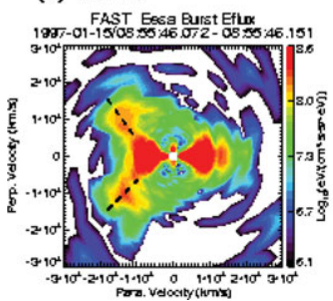

(b) Double shells

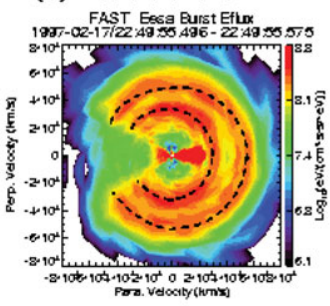

(f) A shell + conic

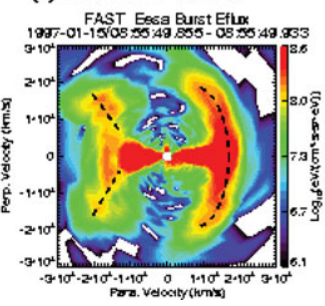

(c) Multiple shells

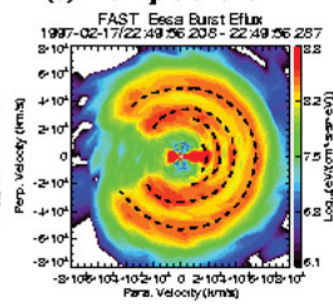

(g) Multiple shellstrings

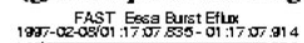

(d) Ring

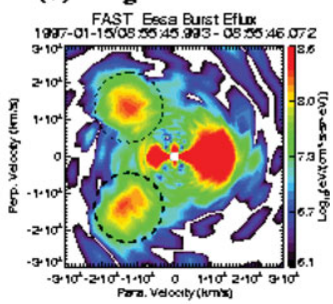

(h) Stable distribution

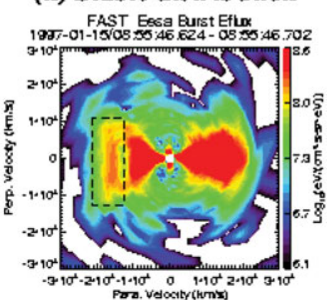

Figure 7. Selected electron distributions observed in the Alfvénic acceleration region by the FAST satellite during S-burst events. The upward direction away from the Earth's ionosphere is to the left (after Su et al. 2008).

\section{Summary}

Since the beginning of space exploration, eight spacecrafts have explored different parts of Jupiter's magnetosphere over last four decades. Seven of these have been flyby missions, including Pioneers 10 (1973) and 11 (1974), Voyagers 1 and 2 (1979), Ulysses (1992), Cassini (2000/2001), and New Horizon (2007). Galileo (1995-2005) was the only orbital mission to date to explore the inner magnetosphere of Jupiter. Many characteristics of plasmas and waves near the Io torus were provided by Io flybys during the Galileo 
mission, however, the plasma environment near Jupiter's polar ionosphere was not adequately sampled. In spite of the lack of a Jovian polar mission, we are able to provide a theoretical understanding of coupling mechanisms between the Io torus and Jupiter's ionosphere based on terrestrial auroral knowledge with confirmation from many Earth satellite missions and ground measurements.

Juno, the second mission in the NASA New Frontiers programs, is currently scheduled for launch in 2011 and to reach Jupiter in 2016 (http://juno.wisc.edu/). Juno's 32 polar orbits will allow for in-situ sampling of Jupiter's auroral acceleration regions. Many of the theoretical/numerical results summarized in this paper will be reexamined in light of new data acquired from this mission. Lessons learned from studies of the JupiterIo interaction, as well investigations of the Earth's auroral region, may be applicable to the Jovian main auroral oval, other less explored magnetized planets, and similar electromagnetic processes of astrophysical sources.

\section{Acknowledgements}

The author thanks Prof. Strassmeier for the invitation to talk about the JupiterIo interaction at IAUS259, Drs. Fran Bagenal, Bob Ergun, and Sam Jones for helpful discussions, and Ron Caton for proofreading the manuscript. She acknowledges support from NASA NNG05GM99G and NSF/CAREER ATM-0544656 to UT Arlington.

\section{References}

Bagenal, F. 2007, J. Atmo. Solar-Terr. Phys. 69, 387

Bagenal, F. 1994, J. Geophys. Res. 99, 11043

Bigg, E. K. 1964, Nature 203, 1008

Chaston, C. C., Carlson, C. W., Peria, W. J., Ergun, R. E., \& McFadden, J. P. 1999, Geophys. Res. Lett. 26, 647

Chust, T., Roux, A., Perraut, S., Louarn, P., Kurth, W. S., \& Gurnett, D. A. 1999, Planet. Space Sci. 47, 1377

Clarke, J. T., Ajello, J., Ballester, G., Jaffel, L. B., Connerney, J., Gérard, J. C., Gladstone, G. R., Grodent, D., Pryor, W., Trauger, J., \& Waite, J. H. 2002, Nature 415, 997

Clarke, J. T., Grodent, D., Cowley, S. W. H., Bunce, E. J., Zarka, P., Connerney, J. E. P., \& Satoh, T. 2004, in: F. Bagenal, T. E. Dowling, \& W. B. McKinnon (eds.), Jupiter: Planet, Satellites, and Magnetosphere, (Cambridge University Press), p. 639

Crary, F. J. 1997, J. Geophys. Res. 102, 37

Crary, F. J., Bagenal, F., Frank, L. A., \& Paterson, W. R. 1998, J. Geophys. Res. 103, 29359

Crary, F. J. \& Bagenal, F. 1997, Geophys. Res. Lett. 24, 2135

Das, A. \& Ip, W.-H 2000, Planet. Space Sci. 48, 127

Delamere, P. A., Bagenal, F., Ergun, R. E., \& Su, Y.-J. 2003, J. Geophys. Res. 108(A6), 1241, doi:10.1029/2002JA009530

Drell, S. D., Foley, H. M., \& Ruderman, M. A. 1965, J. Geophys. Res. 70, 3131

Dols, V. 2001, M.S. Thesis, (Univ. of Alaska, Fairbanks)

Ergun, R. E., Ray, L., Delamere, P. A., Bagenal, F., Dols, V., \& Su, Y.-J. 2008, J. Geophys. Res., in prep.

Ergun, R. E., Su, Y.-J., Andersson, L., Bagenal, F., Delamere, P. A., Lysak, R. L., \& Strangeway, R. J. 2006, J. Geophys. Res. 111, A06212, doi:10.1029/2005JA011253

Ergun, R. E., Su, Y.-J., \& Bagenal, F. 2002, in: H. O. Rucker, M. L. Kaiser, \& Y. Leblanc (eds.), Planetary Radio Emissions V, (Austrian Academy of Sciences Press), p. 271

Ergun, R. E., Carlson, C. W., McFadden, J. P., Mozer, F. S., \& Strangeway, R. J. 2000a, Geophys. Res. Lett., 27,4053

Ergun, R. E., Carlson, C. W., McFadden, Delory, G. T., Strangeway, R. J. \& Pritchett, P. L. 2000b, ApJ 538, 456

Eshleman, V., Tyler, L., Wood, G., Lindal, G., Anderson, J., Levy, G., \& Groft, T. 1979, Science 204,976 
Fjeldbo, G. A., Kliore, A., Seidel, B., Sweetnam, D., \& Cain, D. 1975, A\&A A 39, 91

Fjeldbo, G. A., Kliore, A., Seidel, B., Sweetnam, D., \& Woiceshyn, P. 1976, in: Gehrels (ed.), Jupiter, (University of Arizona Press, Tucson), p. 239

Frank, L. A. \& Paterson, W. R. 1999, J. Geophys. Res. 104, 10345

Gérard, J.-C., Saglam, A., Grodent, D., \& Clarke, J. T. 2006, J. Geophys. Res. 111, A04202, doi:10.1029/2005JA011327

Gérard, J.-C., Gustin, J., Grodent, D., Delamere, P., \& Clarke, J. T. 2002, J. Geophys. Res. 107, doi:10.1029/2002JA009410

Goertz, C. K. 1980, J. Geophys. Res. 85, 2949

Goertz, C. K. \& Deift, P. A. 1973, Planet. Space Sci. 21, 1399

Goldreich, P. \& Lynden-Bell, D. 1969, ApJ 156, 59

Hess, S., Mottez, F., Zarka P., \& Chust, T. 2008, J. Geophy. Res. 113, A03209, doi:10.1029/2007JA012745

Hess, S., Mottez, F., \& Zarka P. 2007, J. Geophy. Res. 112, A11212, doi:10.1029/2006JA012191

Hinson, D. P., Flasar, F. M., Kliore, A. J., Schinder, P. J., Twicken, J. D., \& Herrera, R. G. 1997, Geophy. Res. Lett. 24, 2107

Jones, S. T. \& Su, Y.-J. 2008, J. Geophys. Res., in press

Kivelson, M. G., Bagenal, F., Kurth, W. S., Neubauer, F. M., Paranicas, C., \& Saur, J. 2004, in: F. Bagenal, T. E. Dowling, \& W. B. McKinnon (eds.), Jupiter: Planet, Satellites, and Magnetosphere, (Cambridge University Press), p. 513

Knight, S. 1973, Planet. Space Sci. 21, 741

Lysak, R. L. 1991, J. Geophys. Res. 96, 1553

Mauk, B. H., Anderson, B. J., \& Thorne, R. M. 2002, in: M. Mendillo, A. Nagy, \& J. H. Waite (eds.), Atmospheres in the Solar System: Comparative Aeronomy, (American Geophysical Union), vol. 130, p. 97

Melrose, D. B. \& Dulk, G. A. 1982, ApJ 259, 844

Neubauer, F. M. 1980, J. Geophys. Res. 85, 1171

Paschmann, G., Haaland, S., \& Treumann, R. (eds.) 2003, in: Auroral Plasma Physics, (Kluwer Academic Publishers), p. 93

Piddington, J. H. \& Drake, J. F. 1968, Nature 217, 935

Ray, L. C., Su., Y.-J., Ergun, R. E., Delamere, P. A., \& Bagenal, F. 2008, J. Geophys. Res., in prep.

Saur, J., Neubauer, F. M., Connerney, J. E. P., Zarka, P., \& Kivelson, M. G. 2004, in: F. Bagenal, T. E. Dowling, \& W. B. McKinnon (eds.), Jupiter: Planet, Satellites, and Magnetosphere, (Cambridge University Press), p. 537

Southwood, D. J., Kivelson, M. G., Walker, R. J., \& Slavin, J. A. 1980, J. Geophys. Res., 85, 5959

Stasiewicz, K., Bellan, P., Chaston, C., Kletzing, C., Lysak, R., Maggs, J., Pokhotelov, O., Seyler, C., Shukla, P., Stenflo, L., Streltsov, A., \& Wahlund, J.-E. 2000, Space Sci. Rev. 92,423

Su, Y.-J., Ma, L., Ergun, R. E., Pritchett P. L., \& Carlson, C. W. 2008, J. Geophys. Res. 113, A08214, doi:10.1029/2007JA012896

Su, Y.-J., Ergun, R. E., Jones, S. T., Ergun, R. E., Strangeway, R. J., Chaston, C. C., Parker, S. E., \& Horwitz, J. L. 2007, J. Geophys. Res. 112, A06209, doi:10.1029/2006JA012131

Su, Y.-J., Jones, S. T., Ergun, R. E., Bagenal, F., Parker, S. E., Delamere, P. A., \& Lysak, R. L 2006, J. Geophys. Res. 111, A06211, doi:10.1029/2005JA011252

Su, Y.-J., Jones, S. T., Ergun, R. E., \& Parker, S. E. 2004, J. Geophys. Res. 109, A11201, doi:10.1029/2003JA010344

Su, Y.-J., Ergun, R. E., Bagenal, F., \& Delamere, P. A. 2003, J. Geophys. Res. 108(A2), 1094, doi:10.1029/2002JA009247

Su, Y.-J., Ergun, R. E., Peterson, W. K., Onsager, T. G., Pfaff, R., Carlson, C. W., \& Strangeway R. J. 2001, J. Geophys. Res. 106, 25595

Wright, A. N. 1987, J. Geophys. Res. 92, 9963

Wu, C. S. \& Lee, L. C. 1979, ApJ 230, 621

Zarka, P. 1992, Adv. Space Res. 12, 99 


\section{Discussion}

STRASSmeIER: Would you say that the Io-Jupiter flux tube is considered a "dynamo"?

Su: Yes. The "dynamo" is due to continuous energy exchanges between charged particles and electromagnetic waves. Io's volcanoes provide the plasma source by the ionization and mass loading processes. The electromagnetic energy is generated by the ionization and mass loading processes between Jupiter's rotating magnetic fields and the Io plasma torus. This energy propagates along the field line and accelerates electrons into Jupiter's ionosphere to create aurora emissions.

Blackman: Does the energy in S-bursts, or auroral emission, scale simply with bulk properties of the magnetospheric system, e.g. surface field density in a "back of the envelope" way (e.g. for application to other systems)?

Su: Yes, the S-burst emissions are determined by the electron cyclotron frequency based on the local magnetic field. The largest frequency of S-bursts is $\approx 39 \mathrm{MHz}$, which corresponds to the surface field of $\approx 13 \mathrm{G}$. Radio emissions from other magnetized planets can serve as a good indicator for the planets' surface magnetic field.

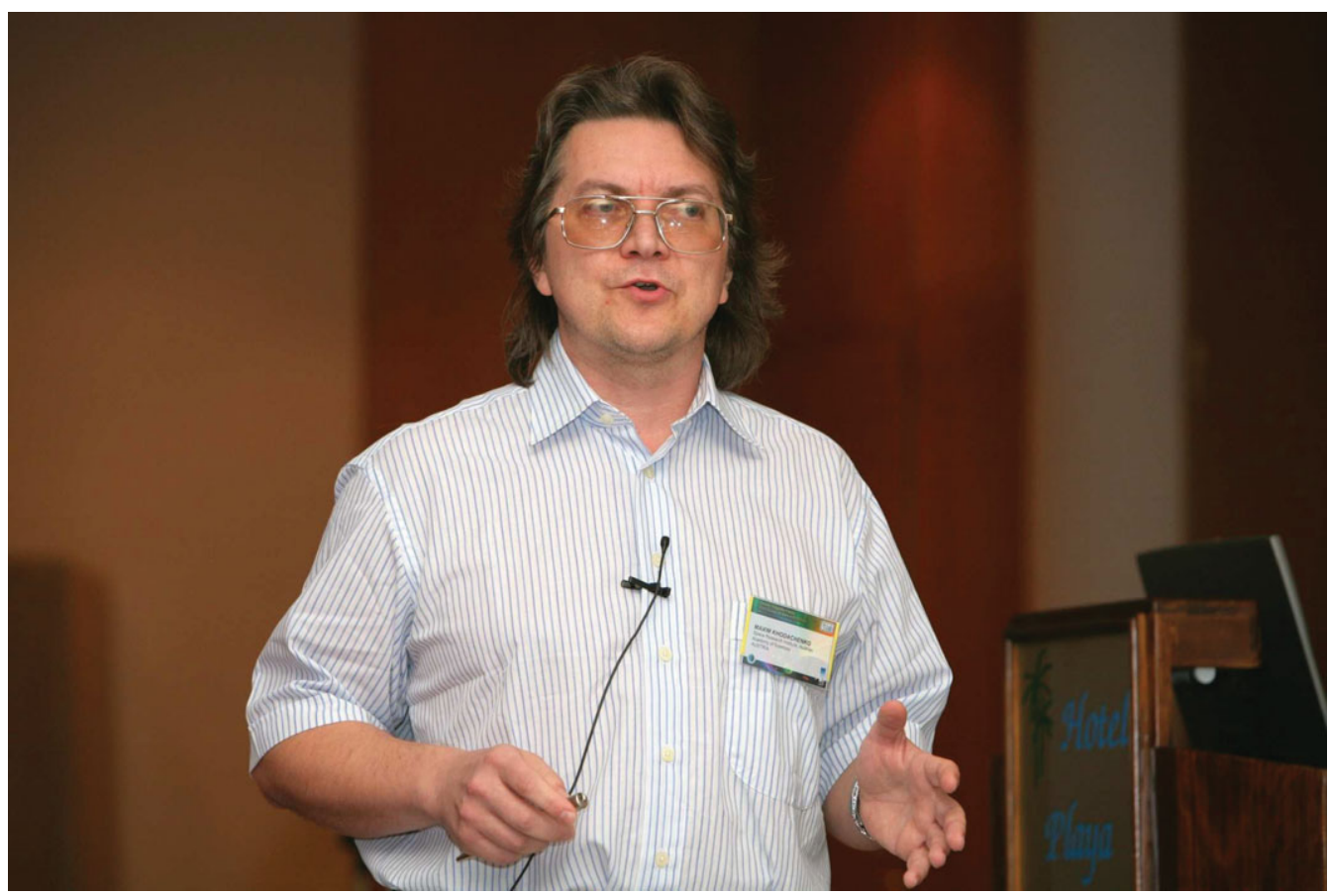

Maxim Khodachenko 